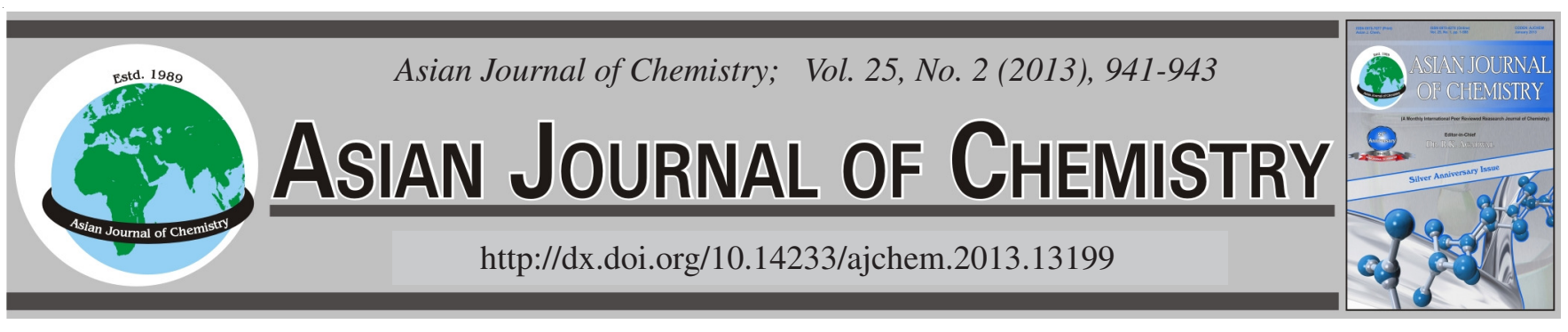

\title{
Synthesis and Spectroscopic Studies of Metal Complexes of Schiff Base Derived from 2-Phenyl-3-( $p$-aminophenyl)-4-quinozolone
}

B.K. Rai ${ }^{1, *}$, S.N. Vidyarathi ${ }^{2}$, Punam Kumari ${ }^{2}$, Suman Kumari ${ }^{2}$, Kumari LaKshmi $^{2}$ and Rajkishore Singh ${ }^{3}$

${ }^{1}$ Department of Chemistry, L.N.T. College, Muzaffarpur-842 002, India

${ }^{2}$ Department of Chemistry, Jai Prakash Vishwavidyalaya, Chapra- 841 301, India

${ }^{3}$ Department of Chemistry, P.R. College, Sonepur-841 101, India

*Corresponding author: E-mail: binodkr_rai@yahoo.co.in

(Received: 7 December 2011;

Accepted: 27 August 2012)

AJC-12015

\begin{abstract}
Ten coordination compounds of $\mathrm{Co}(\mathrm{II}), \mathrm{Ni}(\mathrm{II})$ and $\mathrm{Cu}(\mathrm{II})$ with Schiff base ligand 2-phenyl-3-( $p$-aminophenyl)-4-quinazolone hydrazone $(\mathrm{PAQH})$ were synthesized and characterized by molar mass elemental analyses, magnetic susceptibility, infrared spectra, electronic spectra and molar conductance measurements. All the synthesized compounds were screened for antimicrobial activities against two bacteria, Escherichia coli and Staphylococcus aureus. Metal complexes exhibit several fold increase in their antimicrobial activity in comparison to that of the constituting ligand fragments. The complexes of $\mathrm{Co}(\mathrm{II})$ and $\mathrm{Ni}(\mathrm{II})$ were proposed to be octahedral in geometry whereas the geometry of $\mathrm{Cu}(\mathrm{II})$ complexes were proposed distorted octahedral.
\end{abstract}

Key Words: Schiff base, 2-Phenyl-3-(p-aminophenyl)-4-quinazolone hydrazone, $\mathrm{Co}(\mathrm{II}), \mathrm{Ni}(\mathrm{II}), \mathrm{Cu}(\mathrm{II})$, Complexes.

\section{INTRODUCTION}

The quinazolone derivative have been reported to possess significant biological and pharmacological activities such as anti-HIV ${ }^{1}$, anticonvulsant ${ }^{2}$, antimicrobial ${ }^{3}$, antibacterial ${ }^{4}$, antihypertensive ${ }^{5}$, anthelmintic ${ }^{6}$, antiallergic ${ }^{7}$, analgesic ${ }^{8}$, antiflammatory ${ }^{9}$, insecticidal ${ }^{10}$ and antitubercular ${ }^{11}$. In continuation of our ongoing work ${ }^{11-15}$ on Schiff base transition metal coordination compounds derived from quinazoline, we report here the synthesis and characterization of $\mathrm{Co}$ (II), $\mathrm{Ni}$ (II) and $\mathrm{Cu}(\mathrm{II})$ coordination compounds with 2-phenyl-3-( $p$-aminophenyl)-4-quinazolone hydrazone (PAQH).

\section{EXPERIMENTAL}

All the used chemicals and reagent used were analytical grade. The solvents were used without any purification. The metal contents were determined using standard method ${ }^{16}$. The analytical data of the complexes and their molar conductance values are given in Table-1. The IR spectra of ligand and its complexes were taken on Perkin-Elmer model 577 spectrophotometer using $\mathrm{KBr}$ disc. Conductivity measurements were made using Systronics conductivity meter model 303 in DMF. Magnetic susceptibility were measured by Gouy method using $\mathrm{Hg}\left(\mathrm{Co}(\mathrm{NCS})_{4}\right]$ as a calibrant. Electronic spectra of the complexes were recorded in DMF on Cary-2390 spectrophotometer.
Preparation of ligand: The ligand 3-phenyl-3-( $p$-aminophenyl)-4-quinazolone hydrazone was prepared by condensation of ethanolic solution of 2-phenyl 3-(p-aminophenyl)-4quinazolone $(0.001 \mathrm{~m})$ with hydrazine hydrate $(0.001 \mathrm{~m})$ dissolved in $10 \%$ ethanolic solution.

The resulting mixture was refluxed on waterbath for 3-4 $\mathrm{h}$ with occasional stirring. After cooling the refluxed solution, colourless solid was obtained which was separated by filtration, washed and finally dried and crystallized with tetrahydrofuran. Yield $60 \%$, m.p. $258 \pm 1{ }^{\circ} \mathrm{C}$.

Preparation of the complexes: The complexes of $\mathrm{Co}(\mathrm{II})$, $\mathrm{Ni}(\mathrm{II})$ and $\mathrm{Cu}$ (II) were synthesized by adding ethanolic solution of respective metal acetates $(0.001 \mathrm{~m})$ to Schiff base $(0.002$ $\mathrm{m})$ dissolved in minimum volume of ethanol. The reaction mixture was refluxed on a water bath for $2 \mathrm{~h}$. The solution was then cooled and treated with liquor ammonia/pyridine/ $\alpha$ $\beta$ - or $\gamma$-picoline with each of metal separately and the procedure carried out in each case of similar nature with slight variation of timing of refluxing. The reaction mixture was again refluxed for $2 \mathrm{~h}$. Partial removal of the solvent and cooling to room temperature gave solid coloured complexes which were filtered, washed throughly with ethanol and finally dried.

\section{RESULTS AND DISCUSSION}

The relevant IR spectral bands of ligand as well as metal complexes are listed in Table-2. The IR spectra of the ligand 
TABLE-1

ANALYTICAL AND PHYSICAL MEASUREMENT OF THE COMPLEXES WITH THE LIGAND 2-PHENYL-3 -( $p$-AMINOPHENYL)-4-QUINOZOLONE (PAQH)

\begin{tabular}{|c|c|c|c|c|c|c|c|c|c|c|}
\hline \multirow{2}{*}{ Compounds (Colour) } & \multirow{2}{*}{$\begin{array}{c}\text { Molar } \\
\text { mass }\end{array}$} & \multirow{2}{*}{$\begin{array}{l}\text { Yield } \\
(\%)\end{array}$} & \multicolumn{4}{|c|}{ Elemental analy sis (\%): Found (calcd.) } & \multirow{2}{*}{$\begin{array}{c}\lambda_{\max } \text { electronic } \\
\left(\mathrm{cm}^{-1}\right)\end{array}$} & \multirow{2}{*}{$\mathrm{DT}\left({ }^{\circ} \mathrm{C}\right)$} & \multirow{2}{*}{$\begin{array}{l}\Omega_{\mathrm{m}}\left(\mathrm{ohm}^{-1}\right. \\
\left.\mathrm{cm}^{2} \mathrm{~mol}^{-1}\right)\end{array}$} & \multirow{2}{*}{$\begin{array}{c}\mu_{\mathrm{eff}} \\
(\mathrm{BM})\end{array}$} \\
\hline & & & $\mathrm{M}$ & $\mathrm{C}$ & $\mathrm{H}$ & $\mathrm{N}$ & & & & \\
\hline $\begin{array}{l}\text { PAQH } \\
\text { (Colourless) }\end{array}$ & 251 & 62 & - & $\begin{array}{c}66.74 \\
(66.93)\end{array}$ & $\begin{array}{c}5.09 \\
(5.17)\end{array}$ & $\begin{array}{c}27.76 \\
(27.88)\end{array}$ & - & - & - & - \\
\hline $\begin{array}{l}{\left[\mathrm{Co}(\mathrm{PAQH})_{2}\left(\mathrm{NH}_{3}\right)_{2}\right]} \\
(\text { Brown })\end{array}$ & 592.93 & 63 & $\begin{array}{c}9.84 \\
(9.93)\end{array}$ & $\begin{array}{c}56.48 \\
(56.66)\end{array}$ & $\begin{array}{c}3.96 \\
(4.04)\end{array}$ & $\begin{array}{c}23.50 \\
(23.61)\end{array}$ & 13800,19400 & 203 & 1.4 & 4.93 \\
\hline $\begin{array}{l}{\left[\mathrm{Co}(\mathrm{PAQH})_{2}\left(\mathrm{C}_{6} \mathrm{H}_{5} \mathrm{~N}\right)_{2}\right]} \\
\text { (Brown) }\end{array}$ & 716.93 & 63 & $\begin{array}{c}8.14 \\
(8.21)\end{array}$ & $\begin{array}{c}46.74 \\
(46.86)\end{array}$ & $\begin{array}{c}3.28 \\
(3.34)\end{array}$ & $\begin{array}{c}19.41 \\
(19.52)\end{array}$ & 13300,19900 & 193 & 1.7 & 5.03 \\
\hline $\begin{array}{l}{\left[\mathrm{Co}(\mathrm{PAQH})_{2}(\alpha-\mathrm{pic})_{2}\right]} \\
(\text { Brownish red) }\end{array}$ & 920.93 & 64 & $\begin{array}{c}8.09 \\
(8.17)\end{array}$ & $\begin{array}{c}46.44 \\
(46.60)\end{array}$ & $\begin{array}{c}3.27 \\
(3.32)\end{array}$ & $\begin{array}{c}19.27 \\
(19.41)\end{array}$ & 13600,19700 & 197 & 1.1 & 4.98 \\
\hline $\begin{array}{l}{\left[\mathrm{Co}(\mathrm{PAQH})_{2}(\beta-\mathrm{pic})_{2}\right]} \\
(\text { Brownish red })\end{array}$ & 720.93 & 62 & $\begin{array}{c}8.07 \\
(8.17)\end{array}$ & $\begin{array}{c}46.42 \\
(46.60)\end{array}$ & $\begin{array}{c}3.28 \\
(3.32)\end{array}$ & $\begin{array}{c}19.26 \\
(19.41)\end{array}$ & 13540,20080 & 191 & 0.93 & 4.93 \\
\hline $\begin{array}{l}{\left[\mathrm{Co}(\mathrm{PAQH})_{2}(\gamma-\mathrm{pic})_{2}\right]} \\
\text { (Brownish red) }\end{array}$ & 720.93 & 60 & $\begin{array}{c}8.08 \\
(8.17)\end{array}$ & $\begin{array}{c}46.43 \\
(46.60)\end{array}$ & $\begin{array}{c}3.26 \\
(3.32)\end{array}$ & $\begin{array}{c}19.30 \\
(19.41)\end{array}$ & 13500,19920 & 213 & 2.1 & 4.91 \\
\hline $\begin{array}{l}{\left[\mathrm{Nii}(\mathrm{PAQH})_{2}\left(\mathrm{NH}_{3}\right)_{2}\right]} \\
(\text { Green })\end{array}$ & 592.51 & 62 & $\begin{array}{c}9.81 \\
(9.90)\end{array}$ & $\begin{array}{c}56.55 \\
(56.68)\end{array}$ & $\begin{array}{c}3.97 \\
(4.04)\end{array}$ & $\begin{array}{c}23.53 \\
(23.62)\end{array}$ & $\begin{array}{l}10600,15300 \\
23600\end{array}$ & 218 & 1.4 & 3.17 \\
\hline $\begin{array}{l}{\left[\mathrm{Ni}(\mathrm{PAQH})_{2}(\alpha-\mathrm{pic})_{2}\right]} \\
(\text { Green })\end{array}$ & 720.71 & 63 & $\begin{array}{c}8.03 \\
(8.14)\end{array}$ & $\begin{array}{c}46.50 \\
(46.62)\end{array}$ & $\begin{array}{c}3.26 \\
(3.33)\end{array}$ & $\begin{array}{c}19.36 \\
(19.42)\end{array}$ & $\begin{array}{c}10300,15600 \\
23740\end{array}$ & 189 & 1.9 & 3.22 \\
\hline $\begin{array}{l}{\left[\mathrm{Ni}(\mathrm{PAQH})_{2}(\beta-\text { pic })_{2}\right]} \\
(\text { Deep green })\end{array}$ & 720.71 & 62 & $\begin{array}{c}8.02 \\
(8.14)\end{array}$ & $\begin{array}{c}46.49 \\
(46.62)\end{array}$ & $\begin{array}{c}3.24 \\
(3.33)\end{array}$ & $\begin{array}{c}19.33 \\
(19.42)\end{array}$ & $\begin{array}{c}10270,15800 \\
24000\end{array}$ & 230 & 2.1 & 3.19 \\
\hline $\begin{array}{l}{\left[\mathrm{Ni}(\mathrm{PAQH})_{2}(\gamma \text {-pic })_{2}\right]} \\
(\text { Green })\end{array}$ & 720.71 & 63 & $\begin{array}{c}8.04 \\
(8.14)\end{array}$ & $\begin{array}{c}46.51 \\
(46.62)\end{array}$ & $\begin{array}{c}3.29 \\
(3.33)\end{array}$ & $\begin{array}{c}19.38 \\
(19.42)\end{array}$ & $\begin{array}{c}10240,15300 \\
24600\end{array}$ & 227 & 2.9 & 3.11 \\
\hline $\begin{array}{l}{\left[\mathrm{Cu}(\mathrm{PAQH})_{2}\left(\mathrm{NH}_{3}\right)_{2}\right]} \\
\text { (Blue) }\end{array}$ & 597.54 & 64 & $\begin{array}{c}10.58 \\
(10.67)\end{array}$ & $\begin{array}{c}56.11 \\
(56.23)\end{array}$ & $\begin{array}{c}3.95 \\
(4.01)\end{array}$ & $\begin{array}{c}23.31 \\
(23.42)\end{array}$ & 12200,15000 & 236 & 3.1 & 1.91 \\
\hline $\begin{array}{l}{\left[\mathrm{Cu}(\mathrm{PAQH})_{2}\left(\mathrm{C}_{6} \mathrm{H}_{5} \mathrm{~N}\right)_{2}\right]} \\
\text { (Blue) }\end{array}$ & 720.54 & 62 & $\begin{array}{c}8.04 \\
(8.13)\end{array}$ & $\begin{array}{c}46.44 \\
(46.56)\end{array}$ & $\begin{array}{c}3.26 \\
(3.32)\end{array}$ & $\begin{array}{c}19.26 \\
(19.40)\end{array}$ & 12060,14800 & 224 & 3.4 & 1.89 \\
\hline $\begin{array}{l}{\left[\mathrm{Cu}(\mathrm{PAQH})_{2}(\alpha-\text { pic })_{2}\right]} \\
\text { (Blue) }\end{array}$ & 725.54 & 64 & $\begin{array}{c}8.01 \\
(8.09)\end{array}$ & $\begin{array}{c}46.14 \\
(46.31)\end{array}$ & $\begin{array}{c}3.21 \\
(3.30)\end{array}$ & $\begin{array}{c}19.20 \\
(19.29)\end{array}$ & 11700,14200 & 216 & 3.6 & 1.87 \\
\hline $\begin{array}{l}{\left[\mathrm{Cu}(\mathrm{PAQH})_{2}(\beta-\text { pic })_{2}\right]} \\
\text { (Blue) }\end{array}$ & 725.54 & 63 & $\begin{array}{c}8.02 \\
(8.09)\end{array}$ & $\begin{array}{c}46.11 \\
(46.31)\end{array}$ & $\begin{array}{c}3.24 \\
(3.30)\end{array}$ & $\begin{array}{c}19.18 \\
(19.29)\end{array}$ & 11900,14700 & 196 & 1.1 & 1.85 \\
\hline
\end{tabular}

TABLE-2

IR SPECTRAL BAND $\left(\mathrm{cm}^{2}\right)$ OF THE LIGAND 2-PHENYL-3-( $p$-AMINOPHENYL)-4QUINOZOLONE (PAQH) AND ITS METAL(II) COMPLEXES

\begin{tabular}{lccc}
\hline \multicolumn{1}{c}{ Compounds } & $v(\mathrm{~N}-\mathrm{H})$ & $v(\mathrm{C}=\mathrm{N})$ & $v(\mathrm{M}-\mathrm{N})$ \\
\hline $\mathrm{PAQH}$ & $3400 \mathrm{~s}$, & $1560 \mathrm{~s}, \mathrm{~b}$ & - \\
{$\left[\mathrm{Co}(\mathrm{PAQH})_{2}\left(\mathrm{NH}_{3}\right)_{2}\right]$} & $3370 \mathrm{~m}, \mathrm{~b}$ & $1540 \mathrm{~m}, \mathrm{~b}$ & $450 \mathrm{~m}$ \\
{$\left[\mathrm{Co}(\mathrm{PAQH})_{2}\left(\mathrm{C}_{6} \mathrm{H}_{5} \mathrm{~N}\right)_{2}\right]$} & $3370 \mathrm{~m}, \mathrm{~b}$ & $1535 \mathrm{~m}, \mathrm{~b}$ & $455 \mathrm{~m}$ \\
{$\left[\mathrm{Co}(\mathrm{PAQH})_{2}(\alpha-\mathrm{pic})_{2}\right]$} & $3375 \mathrm{~m}, \mathrm{~b}$ & $1530 \mathrm{~m}, \mathrm{~b}$ & $445 \mathrm{~m}$ \\
{$\left[\mathrm{Co}(\mathrm{PAQH})_{2}(\beta-\mathrm{pic})_{2}\right]$} & $3375 \mathrm{~m}, \mathrm{~b}$ & $1535 \mathrm{~m}, \mathrm{~b}$ & $450 \mathrm{~m}$ \\
{$\left[\mathrm{Co}(\mathrm{PAQH})_{2}(\gamma-\mathrm{pic})_{2}\right]$} & $3375 \mathrm{~m}, \mathrm{~b}$ & $1530 \mathrm{~m}, \mathrm{~b}$ & $435 \mathrm{~m}$ \\
{$\left[\mathrm{Ni}(\mathrm{PAQH})_{2}\left(\mathrm{NH}_{3}\right)_{2}\right]$} & $3370 \mathrm{~m}, \mathrm{~b}$ & $1530 \mathrm{~m}, \mathrm{~b}$ & $430 \mathrm{~m}$ \\
{$\left[\mathrm{Ni}(\mathrm{PAQH})_{2}\left(\mathrm{C}_{6} \mathrm{H}_{5} \mathrm{~N}\right)_{2}\right]$} & $3370 \mathrm{~m}, \mathrm{~b}$ & $1535 \mathrm{~m}, \mathrm{~b}$ & $435 \mathrm{~m}$ \\
{$\left[\mathrm{Ni}(\mathrm{PAQH})_{2}(\alpha-\mathrm{pic})_{2}\right]$} & $3370 \mathrm{~m}, \mathrm{~b}$ & $1535 \mathrm{~m}, \mathrm{~b}$ & $430 \mathrm{~m}$ \\
{$\left[\mathrm{Ni}(\mathrm{PAQH})_{2}(\beta-\mathrm{pic})_{2}\right]$} & $3375 \mathrm{~m}, \mathrm{~b}$ & $1530 \mathrm{~m}, \mathrm{~b}$ & $430 \mathrm{~m}, \mathrm{~b}$ \\
{$\left[\mathrm{Ni}(\mathrm{PAQH})_{2}(\gamma-\mathrm{pic})_{2}\right]$} & $3375 \mathrm{~m}, \mathrm{~b}$ & $1520 \mathrm{~m}, \mathrm{~b}$ & $430 \mathrm{~m}, \mathrm{~b}$ \\
{$\left[\mathrm{Cu}(\mathrm{PAQH})_{2}\left(\mathrm{NH}_{3}\right)_{2}\right]$} & $3370 \mathrm{~m}, \mathrm{~b}$ & $1535 \mathrm{~m}, \mathrm{~b}$ & $430 \mathrm{~m}, \mathrm{~b}$ \\
{$\left[\mathrm{Cu}(\mathrm{PAQH})_{2}\left(\mathrm{C}_{6} \mathrm{H}_{5} \mathrm{~N}\right)_{2}\right]$} & $3370 \mathrm{~m}, \mathrm{~b}$ & $1530 \mathrm{~m}, \mathrm{~b}$ & $420 \mathrm{~m}, \mathrm{~b}$ \\
{$\left[\mathrm{Cu}(\mathrm{PAQH})_{2}(\alpha-\mathrm{pic})_{2}\right]$} & $3375 \mathrm{~m}, \mathrm{~b}$ & $1525 \mathrm{~m}, \mathrm{~b}$ & $425 \mathrm{~m}, \mathrm{~b}$ \\
{$\left[\mathrm{Cu}(\mathrm{PAQH})_{2}(\beta-\mathrm{pic})_{2}\right]$} & $3375 \mathrm{~m}, \mathrm{~b}$ & $1520 \mathrm{~m}, \mathrm{~b}$ & $425 \mathrm{~m}, \mathrm{~b}$ \\
{$\left[\mathrm{Cu}(\mathrm{PAQH})_{2}(\gamma-\mathrm{pic})_{2}\right]$} & $3375 \mathrm{~m}, \mathrm{~b}$ & $1525 \mathrm{~m}, \mathrm{~b}$ & $420 \mathrm{~m}, \mathrm{~b}$ \\
\hline
\end{tabular}

observed strong and broad band at $3400 \mathrm{~cm}^{-1}$ regions In the spectra of the complexes this band shift with slightly reduced intensity. The shift of the band and change in intensity proposing coordination of secondary amino group with metal ion which is also supported by appearance of a band at 460$420 \mathrm{~cm}^{-1}$ assigned $v(\mathrm{M}-\mathrm{N})^{18-20}$. The next IR spectrum of the ligand shows strong band at $1560 \mathrm{~cm}^{-1}$ assigned to $\mathrm{v}(\mathrm{C}=\mathrm{N})$. In the spectra of the complexes this band shifted with slightly reduced intensity. The shift of the band and change in intensity suggest coordination of azomethine nitrogen with metal ion. The low value of molar conductance in the range 2.6 to 4.2 $\mathrm{ohm}^{-1} \mathrm{~cm}^{-2} \mathrm{~mol}^{-1}$ which indicates non electrolytic nature of the complexes. The spectra of pyridine and picolines adducts show additional bands near $650 \mathrm{~cm}^{-1}$ assigned to pyridine and picolin ring. The presence of band at 1480 and $1360 \mathrm{~cm}^{-1}$ with a separation of $120 \mathrm{~cm}^{-1}$ suggests ${ }^{21}$ monocoordinated nature of nitrate group.

The electronic spectral and magnetic susceptibility measurements (Table-1) proposes octahedral geometry for $\mathrm{Co}(\mathrm{II})$ and $\mathrm{Ni}$ (II) complexes. The geometry for $\mathrm{Cu}$ (II) complexes is proposed to distorted octahedral in nature ${ }^{22-25}$.

Antimicrobial screening: The antimicrobial screening of the synthesized Schiff base ligands and their corresponding metal complexes on Staphylococcus aureus (Gram +ve) and Echerichia coli (Gram -ve) by disc diffusion technique ${ }^{26}$ using $\mathrm{DMF}$ as a solvent at concentration of $50 \mathrm{mg}$. On comparing 
the biological activity of the Schiff base and its metal complexes with the standard bacteria, it reflects that the metal complexes have moderate activity as compared to the standard, but all the complexes are more active than ligand due to chelation theory. Chelation tends to make the Schiff base to act as more powerful and potent bacteriostatic agents, thus inhibiting the growth of bacteria and fungi more than the parent Schiff base. The enhancement in biocidal activity may be understood on the basis of their structure that mainly possesses azomethine linkage. Ligands with nitrogen and oxygen donor system inhibit enzyme activity, since the enzymes which require these groups for their activity appear to be especially more susceptible to deactivation. Actually coordination reduces the polarity of the metal ion mainly due to the partial sharing of its positive charge with the donor group within the chelate ring system formed during coordination. This process, in turn, increases the biophilic character of the metal, which favours its permeation more efficiently the lipid layer of the microorganism, thus destroying them more aggressively.

\begin{tabular}{lcc}
\hline \multicolumn{3}{c}{ TABLE-3 } \\
\multicolumn{3}{c}{$\begin{array}{l}\text { MINIMUM INHIBITING CONCENTRATION (MIC) } \\
(\mu \mathrm{g} / \mathrm{mL}) \text { OF THE COMPOUNDS }\end{array}$} \\
\hline \multicolumn{1}{c}{ Compounds } & E. coli & S. aureus \\
\hline PAQH & 40 & 55 \\
{$\left[\mathrm{Co}(\mathrm{PAQH})_{2}\left(\mathrm{NH}_{3}\right)\right]$} & 95 & 86 \\
{$\left[\mathrm{Ni}(\mathrm{PAQH})_{2}\left(\mathrm{NH}_{3}\right)\right]$} & 70 & 75 \\
{$\left[\mathrm{Cu}(\mathrm{PAQH})_{2}\left(\mathrm{NH}_{3}\right)\right]$} & 65 & 65 \\
\hline
\end{tabular}

\section{Conclusion}

The synthesized Schiff base, 2-phenyl-3-( $p$-aminophenyl)4-quinazolone hydrazone (PAQH) acts as uninegative bidentate ligand. The metal ions are coordinated through the amine and imine $\mathrm{N}$ atoms. The remaining coordination centres are satisfied by neutral ligands such as ammonia, pyridine, $\alpha$-picolin, $\beta$ picolin and $\gamma$-picolin. All these observations put together lead us to propose the complexes of $\mathrm{Co}$ (II) and $\mathrm{Ni}$ (II) to have octahedral geometry whereas geometry of $\mathrm{Cu}$ (II) complexes is proposed distorted octahedral geometry as shown in Fig. 1.

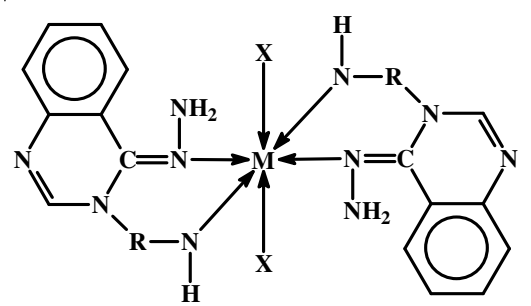

$\mathrm{M}=\mathrm{Co}(\mathrm{II}), \mathrm{Ni}(\mathrm{II})$ and $\mathrm{Cu}(\mathrm{II}) ; \mathrm{X}=\mathrm{NH}_{3}, \mathrm{C}_{6} \mathrm{H}_{5}$, $\alpha$-picoline, $\beta$-picoline and $\gamma$-picoline; $\mathrm{R}=$ Phenyl

Fig. 1. Proposed Structure of $\left[\mathrm{M}(\mathrm{PAQH})_{2} \mathrm{X}_{2}\right]$

\section{REFERENCES}

1. V. Algarsamy, R. Giridhar, M.R. Yadav, R. Revati, K. Ruckmani and E. De. Chercq, Indian J. Pharm. Sci., 69, 304 (2007).

2. A.G.A. El-Helby, Acta Pharm., 53, 127 (2003).
3. N.M. Raghwendra, P.P. Thampi and P.M. Gurubasavarajswamy, E-J. Chem., 5, 23 (2008).

4. S.T. Pai and M.W. Platt, Lett. Appl. Microb., 20, 14 (1994).

5. P.A.J. Janseen, J. Coordiovase Pharmacol., 7, 52 (1985).

6. D.P. Gupta, S. Ahmad, A. Kumar and K. Shankar, Indian J. Chem., 27B, 1060 (1988).

7. B.R. Vogt and L.M. Simpkins, Ger. Pat. Offen, 2,916,992 (1979); Chem. Abstr., 92, 111052 (1980).

8. E. Berenyi, E. Szirt, P. Gorog, L. Petocz, I. Kosoczky, A. Kovacs and G. Urmos, Ger. Pat Offen, 3,0199 (1980); Chem. Abstr., 94, 156963 (1981).

9. E.J. Alexander, U.S. Pat., 4,105,766 (1976); Chem Abstr., 90, 87505 (1979).

10. T. Singh, S. Sharma, V. K. Shrivastava and A. Kumar, Indian J. Chem., 45B, 2558 (2006).

11. S.R. Pattan, V.V.K. Reddy, F.V. Manvi, B.G. Desai and A.R. Bhat, Indian J. Chem., 45B, 1778 (2006).

12. B.K. Rai and K. Sharma, Asian J. Chem., 20, 137 (2008); B.K. Rai, R. Rai, P. Sahi and S. Rana, Asian J. Chem., 20, 143, 149 (2008).

13. B.K. Rai, Z. Hussain, U.P. Singh, S.N. Prasad, A. Prasad and P.M. Mishra, Ultra J. Chem., 4, 53 (2008); B.K. Rai and S.N. Prasad, Ultra J. Chem., 4, 71 (2008); B.K. Rai and A. Kumar, Ultra J. Chem.; 4, 179 (2008); B.K. Rai, J. Indian Coun. Chem., 25, 137 (2008); B.K. Rai, A. Baluni, A. Prasad, R. Thakur and P. Prakash, Asian J. Chem., 21, 3708, 3713 (2009).

14. B.K. Rai and Vinayak, Ultra J. Chem., 5, 67 (2009); B.K. Rai, A. Kumar and Ravishankar, Ultra J. Chem., 5, 73 (2009); B.K. Rai, S. Kumari, R.K. Singh, A. Prasad, M.P. Sinha and P.M. Mishra, Ultra J. Chem., 5, 83 (2009); A. Prasad and B.K. Rai, Orient J. Chem., 25, 175 (2000); B.K. Rai, Ravishankar and S. Pandey, Asian J Chem., 21, 5409, 5994 (2009); B.K. Rai, A. Prasad, Vinayak, A. Kumar and S. Jha 'Sunit', Asian J. Phys., 18, 63 (2009); B.K. Rai, V. Singh, Vinayak, S.P. Singh and S. Jha 'Sunit', Asian J. Phys., 18, 67 (2009); B.K. Rai, J. Indian Coun. Chem., 26, 121 (2009); B.K. Rai, Asian J. Chem., 22, 2761 (2010); B.K. Rai and C. Kumar, Asian J. Chem., 22, 5613 (2010); B.K. Rai and S. Singh, Asian J. Chem., 22, 5619 (2010); B.K. Rai and K.K. Sharma, Asian J. Chem., 22, 5625 (2010).

15. B.K. Rai and R. Kumari, Asian J. Chem., 23, 4625 (2011); B.K. Rai, P. Sinha, S.N. Vidyarathi and V. Singh, Asian J. Chem., 23, 4629 (2011); B.K. Rai, V. Singh, S.N. Vidyarathi and P. Sinha, Asian J. Chem., 23, 4638 (2011); B.K. Rai and B. Kumar, Asian J. Chem., 23, 4635 (2011); B.K. Rai and K.K. Sharma, Orient. J. Chem., 27, 143 (2011).

16. A.I. Vogel, Vogel's Text Book of Quantitative Chemical Analysis, Revised by J. Mendham, R.C. Denny, J.D. Barnes and M. Thomas, Pearson Education (2008).

17. W. Kemp, Organic Spectroscopy, Polgrave, MacMillan Press Ltd, New York, R.M. Silverstein and F.X. Webster, Spectrometric Identification of Organic Compounds, John Wiley \& Sons, edn. 6 (2008).

18. J.R. Ferraro, Low Frequency Vibration of Inorganic and Coordination Compound, Plenum Press, New York.

19. C.N.R. Rao, Chemical Application of IR Spectroscopy, Academic Press, New York (1963).

20. J.A. Wolmsley and S.V. Tyree, Inorg. Chem., 2, 312 (1963); O.N. Sathyanarayn and C.C. Patel, Indian J. Chem., 5, 360 (1967).

21. C.J. Bollhausen, Introduction of Ligand Field Theory, McGraw Hill, New York, p. 208 (1962).

22. C.C. Addison, N. Logan, S.C. Wallwork and D.C. Barner, Quart. Rev. (1971).

23. A.B.P. Lever, Inorganic Electronics Spectroscopy, Elsevier Amsterdam, p. 395 ( 1968).

24. B.N. Figgis, Introduction to Ligand Field, Wiley Eastern Ltd., New Delhi, p. 279 (1976).

25. R.L. Carlin and A.J. Van Dryneveledt, Magnetic Properties of Transition Metal Compounds, Springer Verlag, New York (1997).

26. P.K. Mukherjee, K. Saha, S.N. Giri, M. Pal and B.P. Saha, Indian J. Microbiol., 35, 327 (1995). 